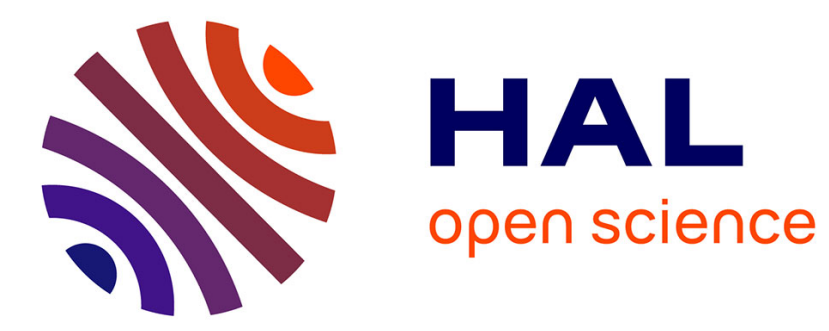

\title{
Color and Spectral Mixings in Printed Surfaces
}

Mathieu Hébert, David Nebouy, Serge Mazauric

\section{To cite this version:}

Mathieu Hébert, David Nebouy, Serge Mazauric. Color and Spectral Mixings in Printed Surfaces. Computational Color Imaging Workshop, Mar 2015, Saint-Etienne, France. pp.3-16, 10.1007/978-3319-15979-9_1. hal-01135956

\section{HAL Id: hal-01135956 https://hal.science/hal-01135956}

Submitted on 26 Mar 2015

HAL is a multi-disciplinary open access archive for the deposit and dissemination of scientific research documents, whether they are published or not. The documents may come from teaching and research institutions in France or abroad, or from public or private research centers.
L'archive ouverte pluridisciplinaire HAL, est destinée au dépôt et à la diffusion de documents scientifiques de niveau recherche, publiés ou non, émanant des établissements d'enseignement et de recherche français ou étrangers, des laboratoires publics ou privés. 


\title{
Color and spectral mixings in printed surfaces
}

\author{
Mathieu Hébert, David Nebouy, Serge Mazauric \\ ${ }^{1}$ Université de Lyon, Université Jean Monnet de Saint-Etienne, CNRS UMR5516 Laboratoire \\ Hubert Curien, 18 rue Benoît Lauras, F-42000 Saint-Etienne, France. \\ mathieu.hebert@univ-st-etienne.fr
}

\begin{abstract}
The present paper discusses the concept of subtractive color mixing widely used in color hardcopy applications and shows that a more realistic concept would be "spectral mixing": the physical description of the coloration of light by printed surfaces comes from the mixing of light components selectively absorbed by inks or dyes during their patch within the printing materials. Some classical reflectance equations for continuous tone and halftone prints are reviewed and considered as spectral mixing laws. The challenge of extending these models to new inkless printing processes based on laser radiation is also addressed.
\end{abstract}

Keywords: Color mixing, printing, halftone colors, spectral reflectance.

\section{$1 \quad$ Introduction}

Color mixing is a key-concept in color reproduction, either by painting, printing, or displaying. It refers to the observation that a large panel of colors (the color gamut) can be achieved by varying the amount of a limited set of base colors, called primaries. With light emitting systems, the primaries are light sources, often with red, green and blue color, that are either superposed or juxtaposed with a shorter period than the visual acuity. Since the tristimulus values of the produced colors is a linear, additive combination of the tristimulus values of the three primaries, this type of color mixing has been called additive color mixing. This concept, based on Grassman's additivity law, enabled the color matching experiments at the basis of colorimetry [1]. In opposition to the light emitting systems, paintings and printed hardcopies selectively attenuate the incident white light in different proportions according to the wavelength. Layers of primaries, paints or inks, are coated on a reflecting support and play a role of spectral filtering of light. This type of color mixing is improperly called subtractive color mixing [2], by reference to the fact that part of the incident light is removed by filtering, but the tristimulus values of paint or ink mixtures cannot be obtained by combining the tristimulus values of the primaries; it is therefore not a color mixing in the sense of colorimetry.

However, the subtractive color mixing is also related to a physical experience, which consists in producing many colors by mixing nonscattering dyes, usually of cyan, magenta and yellow color. According to the Beer-Lambert-Bouguer law [1], the spectral absorption coefficient of the dye mixture, $K(\lambda)$, is a linear, additive combi- 
nation of the spectral absorption coefficients $K_{j}(\lambda)$ of the individual dyes. "Spectral mixing" would therefore be more exact than "color mixing". As the light is exponentially attenuated as a function of the traveled distance in the mixture layer, the internal spectral transmittance of a layer of mixture with thickness $h$ is

$$
t(\lambda)=e^{-K(\lambda) h}=\prod_{j} e^{-c_{j} K_{j}(\lambda) h}
$$

where $c_{j}$ denotes the relative concentration of each dye. By defining a reference concentration of each dye and a reference layer thickness, an internal transmittance $t_{j}(\lambda)$ can be attached to each primary, and the internal transmittance of the mixture can be written

$$
t(\lambda)=\prod_{j} t_{j}^{\varepsilon_{j}}(\lambda)
$$

where $\varepsilon_{j}$ denotes the relative optical thickness of each dye, i.e. the product of its relative concentration and the layer thickness. Note that doubling the amounts of primaries or doubling the layer thickness yields same internal transmittance $t^{2}(\lambda)$, i.e. a double optical thickness. Mixing the dyes or coated them on top of each other would yields exactly the same color.

The Beer-Bouguer-Lambert law is an essential physical law to explain the colors achieved by painting and prints, but not sufficient because no object is a simple mixing or superposition of absorbing media. At least, the mixture has an interface with air whose optical effect cannot be neglected [3-4], and it is generally deposited on a support, specular or diffusing, whose internal reflectance has obviously capital impact of the final color. The refractive index of the layers may also have significant impact, as observed by stacking colored films on a white background with or without optical contact [5-7]. Lastly, the Beer-Bouguer-Lambert law is restricted to non-scattering media; extension to scattering media is possible but more difficult, except when scattering is sufficiently strong so that the Kubelka-Munk model applies [8-9].

Our intension in this paper is to review some spectral reflectance models developed for predicting the color of printed surfaces and present them as so many examples of spectral mixing laws. By recalling some of the main spectral mixing equations that have been physically validated on different types of printed surfaces [10-11], this study extends the recent study aiming at defining different color mixing systems for computer graphics applications [12]. For the sake of simplicity, we address only the case of printing on diffusing supports (paper, white polymer...) with inks assumed to be nonscattering. This already covers most classical printing systems such as woodcut, analog photography, offset, inkjet, laser jet, dye diffusion thermal transfer (D2T2) printing techniques [13]. We also address, in the last section, new inkless printing technologies being currently developed, which produce colors on laser-sensitive layers [14-15]. For the surfaces colored with these new processes, the classical concept of internal transmittance of primary, introduced above in the context of the BeerLambert-Bouguer law and used in most of the models reviewed here, needs to be revisited. 


\section{Spectral mixing laws for contone printing}

Printing systems capable of depositing variable amounts of dyes on the support on each printable pixel, therefore able to produce uniformly colored surfaces as analog photographs, are called continuous tone, or "contone" printing systems. Thermal transfer and thermal diffusion printers belong to this category, especially dye diffusion thermal transfer (D2T2) printers used for proofing applications [13]. On an optical point of view, the structure of the print comprises: a diffusing support, a dye mixture layer, and the dye-air interface. The optical equation proposed in 1953 by Williams and Clapper for photograph prints [16], which have comparable structure, applies. Berns used a simplified version of this equation in 1993 to predict the spectral reflectance of D2T2 prints [17] by ignoring the diffuse or collimated angular distribution of light in the dye layer. The model is based on the spectral internal reflectance $\rho(\lambda)$ of the support and the spectral internal transmittance $t(\lambda)$ of the dye layer assumed to have same refractive index $m$ (typically around 1.5). The reflectance and transmittance of the dye-air interface are computed on both faces according the angular distribution of the light and the measuring geometry: $r_{s}$ denotes the specular reflectance of the interface (it is zero when the specular reflection is not viewed by the detector), $T_{i n}=0.95$ is the transmittance for incoming light at $45^{\circ}, T_{e x}=0.96 / \mathrm{m}^{2}$ is the transmittance for the exiting radiance at $0^{\circ}$ (the term $1 / \mathrm{m}^{2}$ accounts for the effect of the refraction on the radiance [18]), and $r_{i}=0.6$ is the reflectance at the dye side for the light diffuse by the support $[18,19]$. The flux transfers between the support, the dye layer and the interface are presented in Fig. 1.

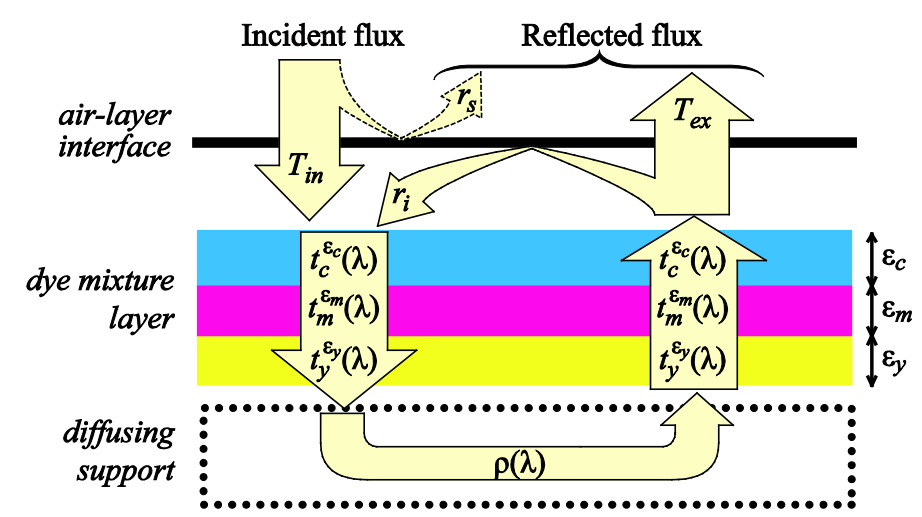

Fig. 1. Path of light in a coloring layer made of three dyes on top of a diffusing support, according to Berns' model.

Finally, Berns equation is written:

$$
R(\lambda)=r_{s}+\frac{T_{i n} T_{e x} \rho(\lambda) t^{2}(\lambda)}{1-r_{i} \rho(\lambda) t^{2}(\lambda)}
$$


When the dye layer is a mixture (or equivalently a superposition) of primary dyes, according to Beer's law (2), the internal transmittance $t(\lambda)$ is given by (2). The support's internal reflectance is deduced from the measured reflectance of the unprinted support. The internal transmittance of each primary, at unit optical thickness (maximum amount of dye), is deduced from the spectral reflectance measured on a color patch where this primary is alone.

\section{Spectral mixing laws for halftone printing}

Most traditional printing systems cannot transfer variable amount of ink on the support but deposit it according to a binary process. Color variations are thus produced by covering partially the surface according to a screen of patterns. this technique is called halftoning [20]. Once printed, the surface looks like a mosaic of colored areas, called Neugebauer primaries, resulting from the partial overlap of the primary ink patterns. For $N$ printed inks, we have $2^{N}$ Neugebauer primaries. In classical clustereddot or error diffusion prints, the fractional area occupied by each Neugebauer primary can be deduced from the surface coverages of the primary inks according to the Demichel equations [21], valid in all cases where the ink halftone dots are laid out independently, e.g. in stochastic screening, in error diffusion, or in mutually rotated clustered dot screens. For three primary inks with respective surface coverages $c, m$, and $y$, the surface coverages $a_{k}$ of the eight primaries are respectively:

$$
\begin{array}{ll}
a_{w}=(1-c)(1-m)(1-y) & a_{m+y}=(1-c) m y \\
a_{c}=c(1-m)(1-y) & a_{c+y}=c(1-m) y \\
a_{m}=(1-c) m(1-y) & a_{c+m}=c m(1-y) \\
a_{y}=(1-c)(1-m) y & a_{c+m+y}=c m y
\end{array}
$$

\subsection{The Yule-Nielsen modified Spectral Neugebauer equation}

The Neugebauer equation is the only additive color mixing law applicable to printed surfaces. It relies on the simple idea that each primary $k$ in the halftone, i.e. each area in the mosaic, contributes to the total reflectance of the surface in proportion of its surface coverage $a_{k}$, and with the same reflectance $R_{k}(\lambda)$ as in a large patch where it is printed alone. The spectral reflectance of a halftone print is thus written [22]

$$
R_{H}(\lambda)=\sum_{k} a_{k} R_{k}(\lambda)
$$

This linear equation can be equivalently expressed in terms of tristimulus values, for example in the CIEXYZ 1931 color space:

$$
\left(X_{H}, Y_{H}, Z_{H}\right)=\sum_{k} a_{k}\left(X_{k}, Y_{k}, Z_{k}\right)
$$

However, the use of the Neugebauer equation is very limited because it does not account for the possible travels of photons through different primaries, especially due 
to the scattering of light by the support. This phenomenon, called optical dot gain or Yule-Nielsen effect [23-24], may have considerable consequence on the spectral reflectance of the printed surface. The Neugebauer model is therefore limited to configurations without any scattering of light (e.g. transparent inks on nonscattering printed supports such as transparency films or mirrors) or where edge effects can be neglected because the primary areas are very large (very low halftone screen frequency).

In order to correct the Neugebauer model, Yule and Nielsen [23] established an empirical law that Viggiano used as a correction of the Neugebauer equation [25], yielding the Yule-Nielsen modified Spectral Neugebauer (YNSN) equation:

$$
R(\lambda)=\left[\sum_{k} a_{k} R_{k}^{1 / n}(\lambda)\right]^{n}
$$

The $n$ value is a real number, usually higher than 1 , which generally increases as the printing support is more scattering or the halftone screen frequency increases [11]. However, it has been noticed that values below 1 or even negative could provide better agreement with the measurements, especially when the ink deeply penetrates the support [26-28].

The physical interpretation of the Yule-Nielsen correction has been explored along various axes, for example by modeling photon path probabilities in the different primaries [29-31]. Recently, an unsuccessful attempt to find other empirical corrections [32] suggests that this correction is the best expression of the physical reality. In most cases, its capacity to match the measured reflectance of halftones is very good, despite its disconcerting simplicity. However, the use of an exponential function in this correction is rather consistent with the physics of attenuation of light in absorbing media.

In addition to the already proposed interpretations, we add a simple one that, as far as we can see, has never been exposed. Let us first schematically consider that the reflection process of light is a succession of two events: the reflection by the support, with reflectance $\rho(\lambda)$, and the attenuation through the halftone ink layer. The reflectance of one Neugebauer primary printed alone is written

$$
R_{k}(\lambda)=\rho(\lambda) T_{k}(\lambda),
$$

where $T_{k}(\lambda)$ can be assimilated to an internal transmittance of the primary, and the Yule-Nielsen modified Spectral Neugebauer equation (7) is written

$$
R(\lambda)=\rho(\lambda)\left[\sum_{k} a_{k} T_{k}^{1 / n}(\lambda)\right]^{n}
$$

If we subdivide the halftone layer into $n$ identical sublayers of relative thickness $1 / n$, the internal transmittance of each one, assuming that no scattering is allowed within it, is

$$
\sum_{k} a_{k} T_{k}^{1 / n}(\lambda)
$$

where the power $1 / n$ comes from the relative thickness $1 / n$ of the sublayer, according to Beer's law. 
The Yule-Nielsen equation (9) means that the light, while crossing the $n$ sublayers, mixes completely between each sublayer as represented in Fig. 2 for $n=2$ : the primaries met by photons through the different sublayers are not correlated. Therefore, the Yule-Nielsen correction actually models the optical dot gain by an alternation of transmissions without scattering through the primaries and complete mixings of the transmitted light components. The $n$ values determines the number of mixing events (which may be extended to real numbers) in the halftone layer, therefore the number of transitions between primaries statistically carried out by the photons. Note that even on transparency films where scattering is very low, the optimal $n$ value is not 1 but rather around $2[4]$.

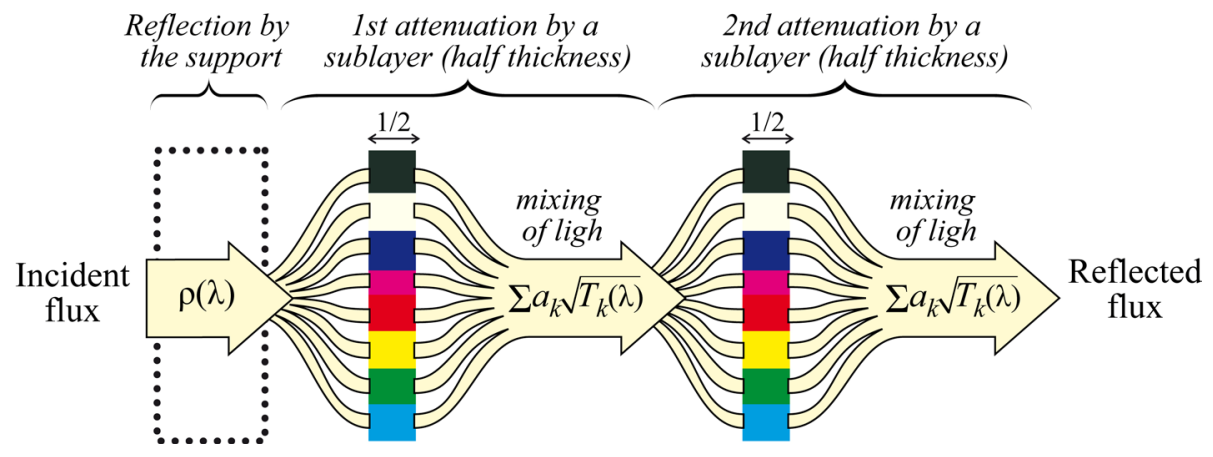

Fig. 2. Physical interpretation of the Yule-Nielsen model for $n=2$.

\subsection{Multiplicative equation for halftone colors}

It is interesting to analyze the limit of the Yule-Nielsen modified Spectral Neugebauer model as $n$ tends to infinity [26-28]. Let us write equation (7) as follows

$$
R(\lambda)=\exp \left[n \log \left(\sum_{k} a_{k} R_{k}^{1 / n}(\lambda)\right)\right]
$$

As $n$ tends to infinity, $1 / n$ tends to 0 and the terms $R_{k}^{1 / n}(\lambda)$ tend to 1 . Since the sum of the surface coverages $a_{k}$ is 1 , the term in the $\log$ function tends to 1 , and the $\log$ function tends to its first order Taylor expansion:

$$
\lim _{n \rightarrow \infty} \log \left(\sum_{k} a_{k} R_{k}^{1 / n}(\lambda)\right)=1-\sum_{k} a_{k} R_{k}^{1 / n}(\lambda)
$$

Using the first order Taylor expansion of the exponential function, we also have

$$
\lim _{n \rightarrow \infty} R_{k}^{1 / n}(\lambda)=\lim _{n \rightarrow \infty} \exp \left(\frac{1}{n} \log R_{k}(\lambda)\right)=\lim _{n \rightarrow \infty}\left[1+\frac{1}{n} \log R_{k}(\lambda)\right]
$$

Finally, we can easily show from (12) and (13) that (11) tends to 


$$
\begin{aligned}
R_{n \rightarrow \infty}(\lambda) & =\lim _{n \rightarrow \infty} \exp \left[n \sum_{k} a_{k}\left(\frac{1}{n} \log R_{k}(\lambda)\right)\right]=\exp \left[\sum_{k} a_{k} \log R_{k}(\lambda)\right] \\
& =\prod_{k} R_{k}^{a_{k}}(\lambda)
\end{aligned}
$$

or, according to the notation introduced in (8), to

$$
R_{n \rightarrow \infty}(\lambda)=\rho(\lambda) \prod_{k} T_{k}^{a_{k}}(\lambda)
$$

This surprising result means that the Yule-Nielsen correction with infinite $n$ transforms the additive Neugebauer equation into a multiplicative one, and the surface coverages into optical thickness as in (2). Pursuing our physical interpretation of the correction, infinite $n$ means infinity of infinitesimal sublayers, which is comparable to a homogenous mixture of the inks for which the Beer-Lambert-Bouguer law applies. This model is particularly suitable to halftones printed on very porous supports such as cotton fabrics, in which the inks deeply penetrate and strongly spread so that the halftone patterns almost completely disappear.

\subsection{The Clapper-Yule equation}

The model introduced in 1953 by Clapper and Yule [33] for the spectral reflectance of single-ink halftone prints follows the same approach as Berns' model for contones with a halftone ink layer in place of the dye mixture layer. The Clapper-Yule equation extended to multi-ink halftones is

$$
R(\lambda)=r_{s}+\frac{T_{i n} T_{e x} \rho(\lambda)\left[\sum a_{k} t_{k}(\lambda)\right]^{2}}{1-\rho(\lambda) r_{i}\left[\sum a_{k} t_{k}^{2}(\lambda)\right]}
$$

where $r_{s}, T_{i n}, T_{\text {out }}, r_{i}$ and $\rho(\lambda)$ have same meaning as in Berns' equation (3).

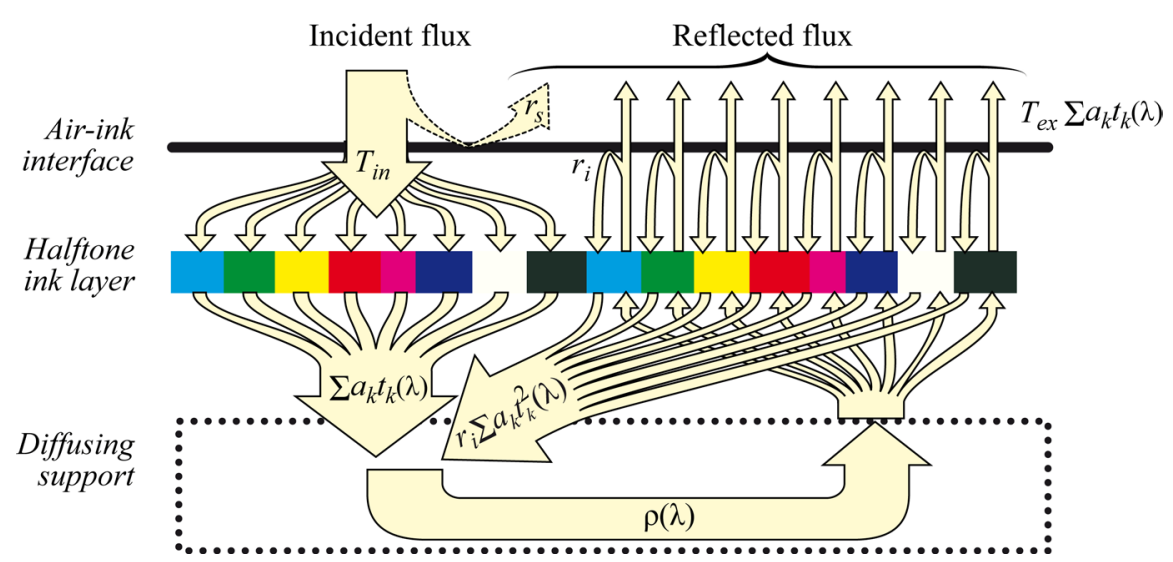

Fig. 3. Path of light in a halftone ink layer on top of a diffusing background, according to the Clapper-Yule model. 
The squared sum in the numerator of (16) denotes the transmissions of the incident light and the exiting light through the halftone ink layer. The sum in the denominator denotes the double attenuation of light issued from the diffusing support that is internally reflected by the interface through each primary, as represented in Fig. 3. The corresponding reflectance is $r_{i} \sum a_{k} t_{k}^{2}$. The diffusing support collects the light components issued from the primaries and mixes them completely.

As for Berns' model, the internal reflectance of the support and the internal transmittance of primaries are deduced from the spectral reflectances measured on the unprinted support and on patches where each Neugebauer primary is printed alone.

Note that both Yule-Nielsen modified Spectral Neugebauer model and the ClapperYule model have been transposed to transmittance, including the possibility to address duplex prints [34-37].

\section{$4 \quad$ Real and virtual primaries}

The previously introduced spectral mixing laws are adapted to classical printing systems where the number of primaries is finite: woodcut printing, offset, inkjet, electrophotography, dye sublimation... [13]. However, they appear to be too limited for new inkless printing technologies based on laser-sensitive layers being currently developed. The concept of primary needs to be reviewed. In order to better understand the challenge of calibrating these printing processes, it is useful to recall how the classical prediction models mentioned above are calibrated.

\subsection{Calibration of models for traditional printing systems}

The reflectances of the primaries in the Yule-Nielsen modified Spectral Neugebauer model, or their internal transmittances in the Berns and Clapper-Yule models, are directly deduced from the measured spectral reflectances of the single-primary color printed on the support. Then, in order to predict the spectral reflectance of any printable color, there is nothing else but getting the quantities (optical thicknesses or, accordingly, surface coverages) of the different primaries that have been actually transferred on the support.

In D2T2 printing, for example, we can verify that, for any printed color, we can find values for the optical thicknesses $\varepsilon_{c}, \varepsilon_{m}$ and $\varepsilon_{y}$ of the three primaries so that the spectral reflectance predicted by (3) and the measured one correctly match. According to our experiments carried out over 54 patches, the deviations between predicted and measured spectra, assessed in terms of CIELAB $1994 \Delta \mathrm{E}$, were 0.43 unit in average, and 1.3 at maximum, which enables validating the accuracy of Berns' equation.

Full calibration of the predictive models also includes the correspondence between the amounts of transferred dyes or printed inks and the CMYK color coordinates of the digital layout. Detailed methods are described in [12] for halftone printing and [17] for contone printing. 


\subsection{Challenges of calibrating inkless printing technologies}

A new generation of inkless printing techniques is currently developed where an achromatic photosensitive layer is coated on a support, then irradiated with lasers in order to reveal the colors. A first example is the laser microinscription system on silvercontaining titania films presented in [14]. Many different angle- and polarizationdependent colors may be produced by varying the laser command parameters (irradiance, wavelength, polarization, exposure...). The concept of primary would need to be extended at least to angle and polarization variations.

Another example is the color laser marking (CLM) system based on the selective bleaching of a mixture of cyan, magenta and yellow dyes under laser irradiation [15]. the bleachable dye mixture is coated on a polymer substrate then overlaid by a clear polymer sheet. The laser irradiation enables bleaching uniformly the dye mixture layer over a large area (see examples in Fig. 4). The structure of the print is comparable to the contone prints and Berns' equation (3) applies.

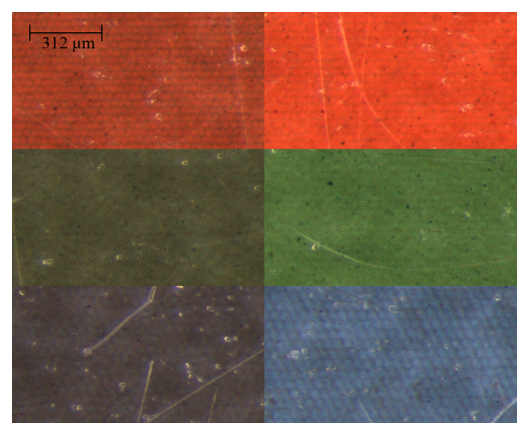

Fig. 4. Microscopic images of areas printed with the CLM technology by using different laser wavelengths and powers. The coloring layer is nearly continuous, no halftoning is needed.

The ideal bleaching process would transform the initial colored dyes into clear ones, but in practice, new color dyes appear due to photo- or thermo-chemical mechanisms. The consequence is that the initial mixture of the three dyes with known internal transmittances is transformed into a mixture containing more dyes with unknown internal transmittances.

We tried to reproduce the classical calibration procedure explained in Section 4.1 for the D2T2 printer, by using samples where each of the three bleachable dyes cyan, magenta and yellow are coated alone. From the spectral reflectances of these samples, we deduced their respective internal transmittances. Then, for 570 other printed patches, we searched for their respective optical thicknesses in order to have the best agreement between the spectral reflectance predicted by the Berns' equation (3) and the measured one. The average CIELAB $1994 \Delta \mathrm{E}$ value over these 570 patches was of 4.2 units, with a maximum of 7.1 units. This poor accuracy shows that this method fails with the CLM printing whereas is was performing for the D2T2 printing. The question is therefore how to obtain effective primaries able to reconstruct the spectral 
space generated by the printing process. One possible solution to get them is to use a principal component analysis (PCA) [17].

\subsection{Mixing virtual primaries obtained by PCA}

We selected a set of 130 patches printed in CLM, measured their spectral reflectances, deduced from them the 130 spectral internal transmittances of transformed dye mixtures, and finally performed the PCA on these 130 internal transmittances. The PCA is computed by creating a rectangular matrix $\mathbf{M}$ whose rows correspond to the spectral internal transmittances, then by diagonalizing the Gramian matrix $\mathbf{M}^{T} \mathbf{M}$ [38-39]. Most eigenvalues of $\mathbf{M}^{T} \mathbf{M}$ are close to zero; the number of eigenvalues with significant value indicates the dimensionality of the spectral space [40], the significance of eigenvalues being assessed by the cumulative percentage variance defined in [38]. The corresponding eigenvectors will be used as the spectral internal transmittances of virtual primaries.

The ten highest eigenvalues obtained from 130 spectral reflectances of CLM patches are shown in Table 1. The cumulative percentage variance reaches $100 \%$ with the four highest eigenvalues: the dimensionality of the spectral space generated by the CLM is therefore 4. By using the four corresponding eigenvectors as internal spectral transmittances in equations (2) and (3), and by searching for their respective optical thicknesses yielding the best agreement between the predicted and measured spectral reflectances for each of the 570 printed patches, we obtained an average CIELAB $\Delta \mathrm{E}_{94}$ value between predicted and measured spectra of 0.55 units (maximum of 1.88 units). With six eigenvectors instead of four, the average $\Delta \mathrm{E}_{94}$ became 0.10 unit (maximum of 0.39 unit). This represents an appreciable gain in accuracy compared to the classical method based on 3 real primaries, and shows that the spectral mixing concept can be extended to "virtual primaries" (containing however all physical information from the measured spectra used in the learning step of the model) when the "real primaries" cannot be clearly identified.

For comparison, we also tested this method from $54 \mathrm{CMY}$ patches printed in D2T2. Compared to CLM printing, similar cumulative variances are achieved with one primary less (Table 1). We can estimate that the dimensionality of the spectral space in D2T2 printing is 3, which is consistent with the fact that three dyes are transferred almost independently of each other [17].

Table 1. Ten highest eigenvalues of the Gramian matrix computed from spectral reflectances of patches printed with the CLM printing process and a D2T2 printer.

\begin{tabular}{lcccccccccc}
\hline CLM printing process & \multicolumn{10}{c}{} \\
\hline Eigenvalue $_{j}$ & 2401 & 65.6 & 14.5 & 4.0 & 0.37 & 0.30 & 0.03 & 0.02 & 0.01 & 0.005 \\
\hline Cumulative \% variance & $\mathbf{9 6 . 6}$ & $\mathbf{9 9 . 2}$ & $\mathbf{9 9 . 8}$ & $\mathbf{1 0 0}$ & 100 & 100 & 100 & 100 & 100 & 100 \\
\hline D2T2 printer & & & & & & & & & & \\
\hline Eigenvalue $e_{j}$ & 529 & 146.4 & 106.0 & 0.12 & 0.11 & 0.03 & 0.01 & 0.008 & 0.002 & 0.001 \\
\hline Cumulative \% variance & $\mathbf{6 7 . 7}$ & $\mathbf{8 6 . 4}$ & $\mathbf{1 0 0}$ & 100 & 100 & 100 & 100 & 100 & 100 & 100 \\
\hline
\end{tabular}




\section{Conclusions}

In this work, we recalled the main predictive equations for the spectral reflectance of printed surfaces: Berns' equation, applicable to contone prints, and the Yule-Nielsen modified Spectral Neugebauer and the Clapper-Yule model applicable to halftone prints, by showing explicitly how they model the spectral attenuations of fluxes in the colored primaries. We espcially reviewed the physical interpretation of the YuleNielsen correction for the Neugebauer equation by showing that it actually models the optical dot gain as an alternation of transmissions of light through the primaries (pure absorption without scattering and transition to other primaries), and complete mixings of the transmitted light components. We also highlighted the two limits of this model according to the value of its tunable parameter $n$ : one limit is the additive Neubaeur equation (only case of additive color mixing applicable to printed surfaces), and the opposite limit is the multiplicative equation where the halftone ink layer tends to become a continuous ink layer. These equations can be considered as spectral mixing laws giving a more physical meaning to the usual concept of subtractive color mixing.

For the new inkless printing technologies based on laser irradiation, these classical models must be extended in order to cope with angle- and polarization dependent colors or to cope with the apparition of unknown primaries without simple correlation with the input parameters of the printing system. However, using a principal component analysis on a large set of printed samples, we can obtain the spectral parameters of a finite number of "virtual primaries" and use them in the classical mixing laws, while keeping satisfying agreement between the predicted and measured spectral reflectances of the printed samples.

\section{Acknowledgement}

This work was supported by the French National Research Agency (ANR) within the program "Investissements d'Avenir" (ANR-11-IDEX-0007), in the framework of project PHOTOFLEX n ${ }^{\circ}$ ANR-12-NANO-0006 and the LABEX MANUTECH-SISE (ANR-10-LABX-0075) of Université de Lyon.

\section{$7 \quad$ References}

1. Wyszecki, G., Stiles, W.S.: Color science: Concepts and methods, quantitative data and formulae, Wiley, New York, 2nd edition (1982).

2. Sharma, G.: Color fundamentals for digital imaging. In Color imaging handbook. CRC Press, New-York (2003).

3. Saunderson, J.L.: Calculation of the color pigmented plastics. J. Opt. Soc. Am. A 32, 727$736(1942)$.

4. Machizaud, J., Hébert, M.: Spectral transmittance model for stacks of transparencies printed with halftone colors. Proc. IS\&T/SPIE Electronic Imaging Symposium, SPIE Vol. 8292, pp. 829240.1-10 (2012) 
5. Simonot, L., Hébert, M., Hersch, R.D.: Extension of the Williams-Clapper model to stacked nondiffusing colored coatings with different refractive indices. J. Opt. Soc. Am. A 23, 1432-1441 (2006).

6. Hébert, M., Hersch, R.D., Simonot, L.: Spectral prediction model for piles of nonscattering films, J. Opt. Soc. Am. A 25, 2066-2077 (2008).

7. Hébert, M., Machizaud, M.: Spectral reflectance and transmittance of stacks of nonscattering films printed with halftone colors. J. Opt. Soc. Am. A 29, 2498-2508 (2012).

8. Kubelka, P.: New contributions to the optics of intensely light-scattering material, part I. J. Opt. Soc. Am. A 38, 448-457 (1948).

9. Emmel, P.: Physical models for color prediction. In Sharma, G, Bala, R. Digital Color Imaging Handbook. CRC Press, New York (2003).

10. Wyble, D. R., Berns, R. S.: A critical review of spectral models applied to binary color printing. Color Res. Appl. 25, 4-19 (2000).

11. Hébert, M., Hersch, R.D.: Review of spectral reflectance prediction models for halftone prints: calibration, prediction and performance. Color Res. Appl.. doi: 10.1002/col.21907 (2014).

12. Simonot, L., Hébert, M.: Between additive and subtractive color mixings: intermediate mixing models. J. Opt. Soc. Am. A 31, 58-66 (2014).

13. Kipphan, H.: Handbook of Print Media. Springer Verlag, Berlin (2001).

14. Crespo-Monteiro, N., Destouches, N., Bois, N., Chassagneux, F., Reynaud, S., Fournel, T.: Reversible and irreversible laser microinscription on silver-Containing mesoporous titania films. Adv. Mater. 22, 3166-3170 (2010).

15. Lutz, N., Zinner. G.: Plastic body, which is provided in the form of a film, for example, a transfer film or laminate film or which is provided with a film of this type, and method for producing color image on or in a plastic body of this type. Patent US2004043308 (2004).

16. Williams, F.C., Clapper, F.R.: Multiple Internal Reflections in Photographic Color Prints. J. Opt. Soc. Am. 43, 595-597 (1953).

17. Berns, R.S.: Spectral modeling of a dye diffusion thermal transfer printer. J. Electron. Imaging 2, 359-370 (1993).

18. Hébert, M., Hersch, M.: Classical Print Reflection Models: A Radiometric Approach, J. Im. Sci. Technol. 48, 363-374 (2004).

19. Judd, D.B.: Fresnel reflection of diffusely incident light. Journal of the National Bureau of Standards 29, 329-332 (1942).

20. Kang, H.R.: Digital color halftoning. SPIE Publications, Washington (1999).

21. Demichel, M.E.: Procédés 26, 17-21 (1924).

22. Neugebauer, H.E.J.: Die theoretischen Grundlagen des Mehrfarbendrucks. Zeitschrift fuer wissenschaftliche Photographie 36, 36-73 (1937). Translated into English: The theoretical basis of multicolour letterpress printing. Color Res. App. 30, 322-331 (2005).

23. Yule, J.A.C., Nielsen, W.J.: The penetration of light into paper and its effect on halftone reproduction. Proc. TAGA 3, 65-76 (1951).

24. Ruckdeschel, F.R., Hauser, O.G.: Yule-Nielsen effect in printing: a physical analysis. Appl. Opt. 17, 3376-3383 (1978).

25. Viggiano, J.A.S.: The Color of Halftone Tints, Proc. TAGA, 647-661 (1985).

26. Lewandowski, A., Ludl, M., Byrne, G., Dorffner, G.: Applying the Yule-Nielsen equation with negative n. J. Opt. Soc. Am. A 23, 1827-1834 (2006).

27. Viggiano, J.A.S.: Ink Penetration, Isomorphic Colorant Mixing, and Negative Values of Yule-Nielsen n. Proc. IS\&T 18th Color and Imaging Conference (Springfield, VA), 285290 (2010). 
28. Viggiano, J.A.S.: Physical Significance of Negative Yule-Nielsen n-value. Proc. IS\&T International Congress of Imaging Sciences (Rochester, NY), 607-610 (2006).

29. Arney, J.S. A probability description of the Yule-Nielsen effect, I: Tone reproduction and image quality in the graphic arts. J. Im. Sci. Technol. 41, 633-636 (1997).

30. Arney, J.S., Kutsube, M.: A probability description of the Yule-Nielsen effect. II : The impact of halftone geometry : Tone reproduction and image quality in the graphic arts. Recent Progress in Digital Halftoning II 41, 637-642 (1999).

31. Ruckdeschel, F.R., Hauser, O.G.: Yule-Nielsen effect in printing: a physical analysis. Appl. Opt. 17, 3376-3383 (1978).

32. Hébert, M.: Yule-Nielsen effect in halftone prints: graphical analysis method and improvement of the Yule-Nielsen transform. Proc. SPIE 9015, Color Imaging XIX: Displaying, Processing, Hardcopy, and Applications, (San Francisco, CA) 90150R (2014).

33. Clapper, F.R., Yule, J.A.C.: The Effect of Multiple Internal Reflections on the Densities of Halftone Prints on Paper. J. Opt. Soc. Am. 43, 600-603 (1953).

34. Hébert, M., Hersch, R.D.: Reflectance and transmittance model for recto-verso halftone prints. J. Opt. Soc. Am. A 23, 2415-2432 (2006).

35. Hébert, M., Hersch, R.D.: Reflectance and transmittance model for recto-verso halftone prints: spectral predictions with multi-ink halftones. J. Opt. Soc. Am. A 26, 356-364 (2009).

36. Mazauric, S., Hébert, M., Simonot, L., Fournel T.: Two-flux transfer matrix model for predicting the reflectance and transmittance of duplex halftone prints. J. Opt. Soc. Am. A 31, 2775-2788 (2014).

37. Hébert, M., Hersch, R.D.: Yule-Nielsen based recto-verso color halftone transmittance prediction model. Applied Optics 50, 519-525 (2011).

38. Tzeng, D.Y., Berns, R.S. A review of principal component analysis and its applications to color technology. Color Research \& Application 30, 84-98 (2005).

39. Bugnon, T.: Flexible and Robust Calibration of the Yule-Nielsen Model for CMYK Prints. PhD Dissertation, Ecole Polytechnique Fédérale de Lausanne, Switzerland (2011).

40. Hardeberg, J.Y.: On the spectral dimensionality of object colours. Proc. IS\&T Conference on Colour in Graphics, Imaging, and Vision (CGIV2002), 480-485 (2002). 